\title{
Long-term follow-up of optic neuritis associated with meningoencephalitis of unknown etiology in a Maltese dog
}

\author{
Sun-Jun Jung ${ }^{1}$, Jury Kim ${ }^{1,2}$, Caryn E Plummer ${ }^{3}$, Ki-Chang Lee ${ }^{1}$, Min-Su Kim ${ }^{4, *}$ \\ ${ }^{1}$ College of Veterinary Medicine, Chonbuk National University, Iksan 54596, Korea \\ ${ }^{2}$ Bundang Bright Eye Animal Hospital, Seongnam 13439, Korea \\ ${ }^{3}$ Department of Small Animal Clinical Science, College of Veterinary Medicine, University of Florida, Gainesville, \\ FL 32610, USA \\ ${ }^{4}$ Department of Veterinary Clinical Science, College of Veterinary Medicine and Research Institute for Veterinary Science, \\ Seoul National University, Seoul 08826, Korea
}

\begin{abstract}
A 6-year-old intact male Maltese dog presented with a history of blindness and ataxia. Neuro-ophthalmic examination revealed dilated pupils with absent pupillary light reflexes and menace response in both eyes. Mild peripapillary edema was noted in the fundus of the right eye. After magnetic resonance imaging, the dog was provisionally diagnosed with meningoencephalitis of unknown etiology. Follow-up funduscopy was performed to monitor the condition of the optic discs for three years. Despite of the treatment with prednisolone, the optic nerve progressed to atrophy and the dog couldn't restore vision.
\end{abstract}

Keywords: dog, fundus, long-term follow-up, meningoencephalitis of unknown etiology, optic neuritis

*Corresponding author

Min-Su Kim

Department of Veterinary Clinical Science, College of Veterinary Medicine and Research Institute for Veterinary Science, Seoul National University, 1 Gwanak-ro, Gwanak-gu, Seoul 08826, Korea

Tel: $+82-2-880-1252$

Fax: $+82-2-887-1213$

E-mail: minsukim@snu.ac.kr

ORCID

Sun-Jun Jung

https://orcid.org/0000-0001-8259-9149

Min-Su Kim

https://orcid.org/0000-0002-7467-496X

Conflict of Interest

The authors declare no conflicts of interest.

Received: January 5, 2019

Revised: March 7, 2019

Accepted: May 30, 2019
Optic neuritis is a disease that causes demyelination of the optic nerve resulting in sudden loss of sight in one or both eyes [1,2]. There are numerous etiologies of optic neuritis such as inflammation, infection, neoplasm, trauma etc. In many cases, however, it is difficult to clarify the cause of optic neuritis. Therefore, most cases are classified as idiopathic [1,3]. Clinical signs of optic neuritis include acute loss of vision and a dilated and unresponsive pupil in the affected eye. On funduscopic examination, the optic disc appears to be swollen and edematous, with blurring of the disc margins. The physiologic cup may be lost as well and the blood vessels of the disc surface are raised, and hemorrhages may be present [1]. Papillitis, peripapillary retinal edema and neuroretinitis are commonly associated with optic neuritis in dogs [2]. The funduscopic examination results may initially be normal if the optic papilla is not involved, but the retrobulbar optic nerve is affected [1,4]. On magnetic resonance imaging (MRI), hyperintensity and thickening of the optic nerve may be observed [3]. Although optic neuritis is controlled properly, it usually progresses to optic nerve atrophy, which appears as a pale, grayish, shrunken optic disc with attenuation of retinal blood vessels. Even with treatment, the prognosis for recovery of vision in cases of optic neuritis is considered to be poor [3].

Meningoencephalitis of unknown etiology (MUE) is a broad term referring to inflammatory lesions in the central nervous system (CNS) that are not supported histologically [5]. MRI and cerebrospinal fluid (CSF) analysis can be used to rule out other inflammatory non-infectious CNS diseases. Depending on the pattern of inflammation and the type of inflammatory cells in tissue samples, specific class of inflammatory brain diseases, such as granulomatous meningoencephalitis (GME), necrotizing meningoencephalitis, and necrotizing leukoencephalitis could be distinguished [6].

In this case, the ophthalmoscopic changes of the optic disc over time in a dog with optic neuritis associated with MUE were documented under the owner's consent. 


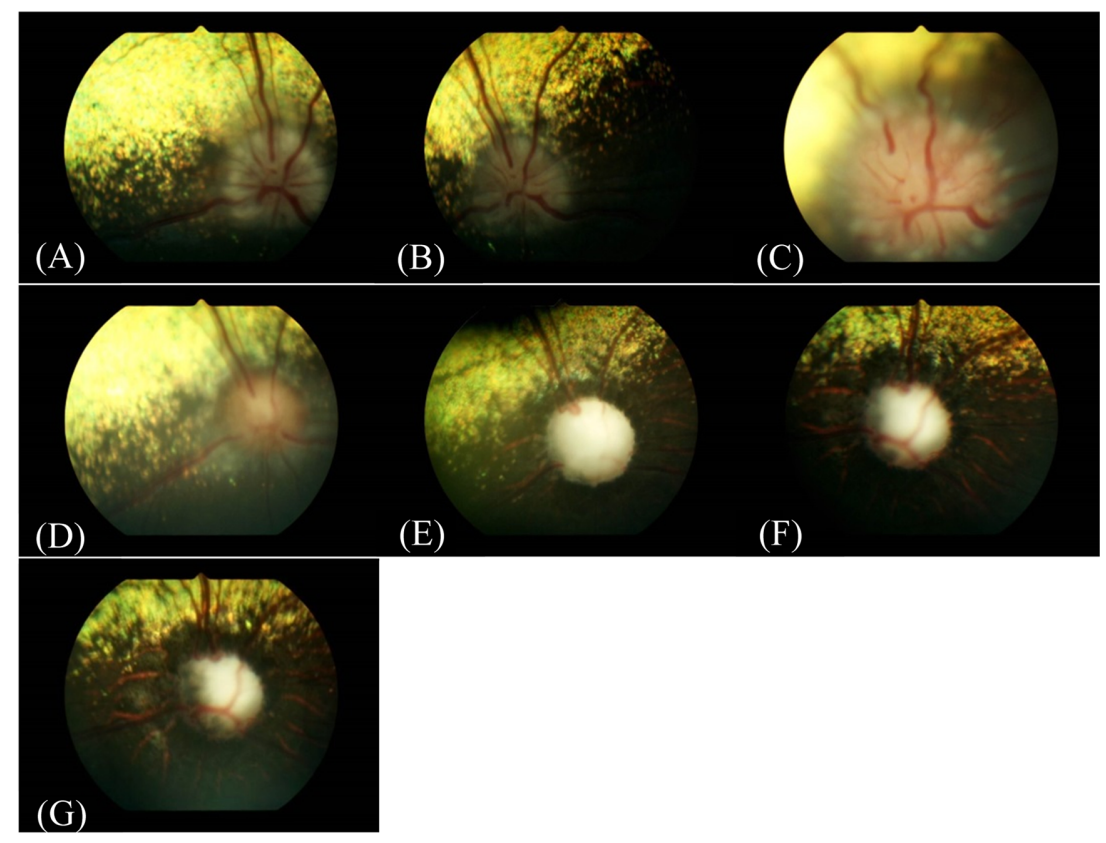

Fig. 1. Serial fundus images of right eye. (A) Day 0, (B) after 1 month, (C) after 2 months, (D) after 3 months, (E) after 18 months, (F) after 31 months, and $(\mathrm{G})$ after 36 months (times in relation to the initial visit).

A six-year-old intact male Maltese dog presented to the Animal Medical Center with a history of cervical pain, forelimb ataxia, and blindness. Neuro-ophthalmic examination revealed dilated pupils with loss of pupillary light reflex (PLR), dazzle reflex, and menace response in both eyes (OU). Intraocular pressure and Schirmer tear test measurements were within normal limits. On funduscopy using a hand-held retinal camera (Genesis-D; Kowa, Japan), the optic disc of the right eye (OD) was observed to have indistinct margins associated with peripapillary edema (Fig. 1A). No abnormalities were observed in the left eye (OS) (Fig. 2A). Electroretinogram (ERG) could not be performed because of the owner's financial difficulties. Findings from blood analysis were within normal limits. Blood serum titres for Ehrlichia canis, Anaplasma phagozytophilia, Toxoplasma gondii and canine distemper virus were negative.

On MRI examination, the right forebrain showed hyperintensity in T2-weighted transverse images (Fig. 3). There were no remarkable findings related to the optic nerve, optic chiasm, or optic tract. In addition, cytologic and biochemical evaluation of CSF obtained from the atlanto-occipital space revealed no increased cell numbers, protein or glucose levels. The dog was tentatively diagnosed with MUE. The dog was treated with prednisolone $(1.0 \mathrm{mg} / \mathrm{kg}$ per orally [PO], twice a day [BID]; Yuhan, Korea) for 7 days. One month after the first visit, menace response and PLR were still absent OD. The optic disc OD was less swollen than at the initial examination (Fig. 1B). However, menace response and PLR were present and vision was restored OS. No abnormalities could be observed in the optic disc OS (Fig. 2B). Cervical pain and forelimb ataxia were resolved. The dosage of prednisolone was reduced $(0.5 \mathrm{mg} / \mathrm{kg}$ PO, BID for 14 days and then $0.2 \mathrm{mg} / \mathrm{kg}$ PO, BID for another 14 days). Two months after the first visit, PLR and menace response were lost OU. The optic disc OD became hyperemic and swollen, and multiple white, circular subretinal infiltrates adjacent to the disc were observed (Fig. 1C). The optic disc OS also became swollen and hyperemic and the dorsal retinal vein became tortuous when compared to the last visit (Fig. 2C). An increased dose of prednisolone (1.0 mg/kg PO, BID) was prescribed for 14 days. Three months after the first visit, the infiltrates disappeared from the border of the optic disc OD. However, the optic disc OD remained hyperemic and the size of the optic disc OD decreased (Fig. 1D). The size of the optic disc OS also decreased and mild peripapillary edema persisted (Fig. 2D). Prednisolone was prescribed as following doses; $1.0 \mathrm{mg} / \mathrm{kg}$ PO, BID for 7 days and then $0.7 \mathrm{mg} / \mathrm{kg}$ PO, BID for 14 days. Even though the medication was continued, vision was not regained. Eighteen months after the initial diagnosis, the optic disc OD was markedly pale relative to its appearance when optic nerve edema recurred, and overt atrophy of the disc had begun in the inferiotemporal region on the border of the optic disc. Depigmentation was observed in the nontapetal fundus (Fig. 1E). In addition, choroidal vessels were visible in the nontapetal fundus due to retinal thinning OU (Figs. 1E and 2E). Systemic therapy with prednisolone $(1.0 \mathrm{mg} / \mathrm{kg}$ PO, BID for 14 days and then 0.5 $\mathrm{mg} / \mathrm{kg}$ PO, BID for 7 days) was prescribed. Thirty-one months post-diagnosis, atrophy of the inferiotemporal region of the optic disc had advanced more centrally OD (Fig. 1F), and severe optic disc atrophy OS could be observed (Fig. 2F). Thirty-six months after the first visit, optic disc atrophy had 


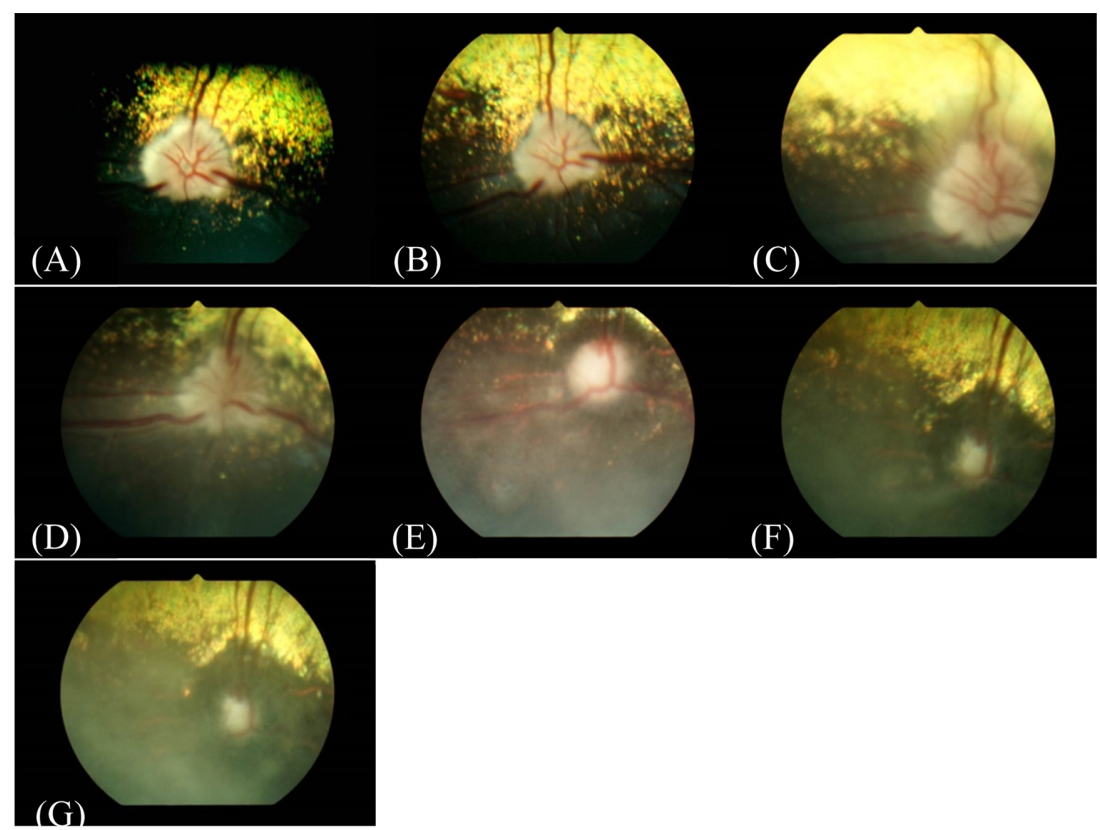

Fig. 2. Serial fundus images of left eye. (A) Day 0, (B) after 1 month, (C) after 2 months, (D) after 3 months, (E) after 18 months, (F) after 31 months, and (G) after 36 months (times in relation to the initial visit).

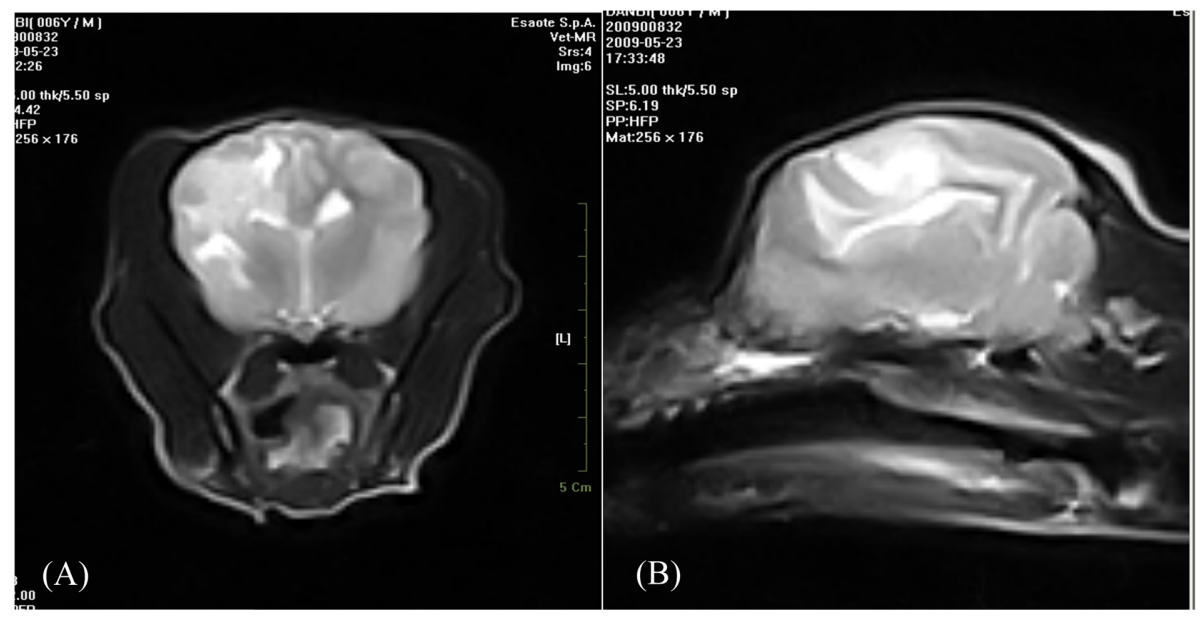

Fig. 3. T2-weighted (A) transverse and (B) sagittal images at a level rostral to the interthalamic adhesion. Note the hyperintense region in the right forebrain.

advanced to the ventral region OD (Fig. 1G). The dosage of prednisolone was increased to $1.5 \mathrm{mg} / \mathrm{kg}$ PO, BID for 7 days. Since corticosteroids were used intermittently for a long period of time without any steady use, no critical side effects were found except mild polyuria and polydipsia. Regular follow-up was difficult because the owner did not come to the hospital regularly. In addition, due to the owner's financial difficulties, relatively expensive immune suppressants could not be used. Over the course of three years following the diagnosis, severe optic atrophy developed OU. The patient was ultimately lost to follow-up after three years. However, phone contact with the owner confirmed that the patient died soon after the last visit. The autopsy could not be carried out because the time had already passed.
The optic nerve consists of the axons of the retinal ganglion cells. The axons exit the eyeballs through the lamina cribrosa and merge to form the retrobulbar optic nerve [2]. Depending on the location of the lesion, optic neuritis may be divided into optic papillitis and retrobulbar neuritis [1]. In this case, optic neuritis was seen as optic papillitis with a swollen optic nerve head and peripapillary edema OD. In OS, the optic disc appeared normal until two months after the initial presentation, although the neuro-ophthalmic examination was abnormal and the eye was non-visual. This implies that the retrobulbar neuritis extended anteriorly to the optic disc or that there was retrograde degeneration of the axons of the optic nerve. In both humans and animals, the cause of the optic neuritis is often difficult to determine [2]. Based on the 
patient's history, the results of ophthalmic and neurologic examinations, clinical pathology, and advanced imaging, the patient was tentatively diagnosed with MUE in this case study. GME is a non-suppurative inflammatory disease of the CNS with unknown etiology, which is one of the classes of MUE [4]. Depending on the location and the pathological and clinical features, GME is classified into focal, disseminated and ocular form. Of these three forms, the ocular form is the least common $[4,7]$. The optic nerve is surrounded by meninges and subarachnoid space. Therefore, diseases of the meninges can affect the optic nerve [2]. Because the ocular form of GME involves the optic nerve, it presents with acute onset of vision loss and unresponsiveness to light stimulation $[4,7]$. On Funduscopic examination, swelling of the optic nerve head, indistinct disc margins, dilated vessels, and, occasionally, retinal hemorrhage or detachment could be revealed. $[1,4]$. Although the precise etiology was undetermined, GME is presumed as the most likely differential diagnosis. If this case definitively diagnosed as GME, it would likely have been considered the ocular form. Changes to the optic disc, including the peripapillary infiltrates, could be observed in serial fundic examinations over time. It is not possible to precisely identify the composition of the infiltrates without histopathologic examination. However, it has been demonstrated previously that histiocytic cells form cuffs around blood vessels and that reticulin fibers form around individual histiocytic cells [8]. A similar case was characterized by granulomatous inflammation around optic nerves in a miniature dachshund with optic neuritis associated with GME [9].

Another interesting finding in our case was the asymmetric manner in which the optic nerve atrophied. The inferiotemporal aspect of the optic disc began to atrophy in OD before the rest of the disc. This may have occurred because the retinal nerve fiber layer (RNFL) is thinnest in the inferior quadrant, followed by the temporal quadrant [10]. One of the main consequences of optic neuritis is axonal loss leading to RNFL thinning and decreased visual acuity. Significant reductions in RNFL thickness are also found in humans with optic neuritis. The thinnest part of the RNFL is the temporal RNFL in humans. It has been reported that the first site to be affected by optic nerve atrophy is the temporal RNFL [11]. However, one previous study reported that RNFL thickness was reduced in the superior, inferior, and nasal quadrants, but was unchanged in the temporal quadrant in a human eye with optic neuritis when compared to the contralateral normal eye [12]. Further studies in normal dogs and dogs with optic neuritis are required to determine whether there truly are differences in the thickness of the RNFL and what changes occur due to diseases of the optic nerve.

Dogs affected with sudden acquired retinal degeneration syndrome (SARDS) also present for sudden vision loss [1315]. It was reported that photoreceptor outer segments of the SARDS affected eyes were lost [14]. As a result, extinguished ERG response is appeared [13-15]. However, on fundus examination, the ocular fundus appears normal $[13,15]$. Decreased
ERG response with normal fundus is the typical feature of the SARDS and it could exclude other causes of acute vision loss, such as optic neuritis, which generally shows normal ERG response $[1,13,16]$. SARDS shows mydriatic pupils with slow response to bright white light. On chromatic PLR, it is responsive to blue light but unresponsive to red light [13]. Optic neuritis also shows mydriasis, however, the pupils are fixed and unresponsive to the lights including white, blue and red [2,3]. Although ERG was not performed in this case, SARDS could be excluded from PLR and the abnormal fundus finding, which is not related to SARDS. However, abnormality of the ocular fundus such as hyper-reflective tapetal fundus, atrophy of the optic nerve head, and attenuation of the retinal vasculature could be found on SARDS affected eyes several months after the onset [16]. In this case, abnormal fundus findings were found in the early stages of vision loss and this could support the fact that it was not SARDS.

The prognosis for vision in optic neuritis is generally regarded to be poor $[1,2,4]$. Several therapies for optic neuritis and MUE have been reported. Immunosuppressive therapy especially corticosteroids, is commonly used [4]. While corticosteroids are the mainstay of immunosuppressive therapy for presumptive GME, response to steroid monotherapy is variable and it is often temporary [17]. Several alternative immunosuppressive drugs evaluated as adjunctive treatment options for GME patients include procarbazine, cytosine arabinoside, and cyclosporine [3,5,17]. Systemic immunosuppressant therapy is routinely used in hopes of enhancing visual recuperation and retention, however, there is no substantive evidence that long-term visual function is effectively maintained with therapy [4]. Optic neuritis in this case was initially responsive to prednisolone, however relapse occurred. Not only the low efficacy of prednisolone, but also the irregularity of the days of dosing and visit intervals could have resulted in poor prognosis.

It has been reported that the survival time of GME cases with focal lesion that were given corticosteroids alone was 41 days ( 3 to $>1215$ days) [17]. In addition, Kitagawa et al reported survival time of 105 days after the onset of the symptoms of ocular GME [9]. Compare to the previous reports, this case had a longer survival time over three years.

Unfortunately, it was impossible to confirm a final etiologic diagnosis in this case because the patient was lost to follow-up after three years of treatment. Because a necropsy was not conducted, a histological diagnosis could not be made. This case is, however, of great clinical significance, as it documents the pathologic progression of optic neuritis associated with MUE by serial funduscopy over three years.

\section{Acknowledgements}

This work was supported by the National Research Foundation of Korea (NRF) grant funded by the Korea government (MSIT) (No. 2019R1F1A1063220). 


\section{References}

1. Adamo PF, Adams WM, Steinberg H. Granulomatous meningoencephalomyelitis in dogs. Compend Contin Educ Vet 2007;29:678-690.

2. Costello F, Hodge W, Pan YI, Burton JM, Freedman MS, Stys PK, Trufyn J, Kardon R. Sex-specific differences in retinal nerve fiber layer thinning after acute optic neuritis. Neurology 2012;79:1866-1872.

3. Dubielzig RR, Ketring KL, McLellan GJ, Albert DM. Veterinary Ocular Pathology E-Book: a Comparative Review. Elsevier Health Sciences, Oxford, 2010.

4. Fischer CA, Jones GT. Optic neuritis in dogs. J Am Vet Med Assoc 1972;160:68-79.

5. Hernandez-Merino E, Kecova H, Jacobson SJ, Hamouche KN, Nzokwe RN, Grozdanic SD. Spectral domain optical coherence tomography (SD-OCT) assessment of the healthy female canine retina and optic nerve. Vet Ophthalmol 2011; 14:400-405.

6. Kitagawa M, Okada M, Watari $T$, Sato T, Kanayama K, Sakai T. Ocular granulomatous meningoencephalomyelitis in a dog: magnetic resonance images and clinical findings. $\mathrm{J}$ Vet Med Sci 2009;71:233-237.

7. Komáromy AM, Abrams KL, Heckenlively JR, Lundy SK, Maggs DJ, Leeth CM, MohanKumar PS, Petersen-Jones SM, Serreze DV, van der Woerdt A. Sudden acquired retinal degeneration syndrome (SARDS): a review and proposed strategies toward a better understanding of pathogenesis, early diagnosis, and therapy. Vet Ophthalmol 2016;19:319-331.

8. Maehara T, Shimada A, Morita T, Sawashima Y, Sawashima K. Distribution of the inflammatory lesions in the central nervous system of dogs affected with disseminated and ocular form of granulomatous meningoencephalomyelitis. J Vet Med Sci 2009;71:509-512.
9. Maggs DJ, Miller P, Ofri R. Slatter's Fundamentals of Veterinary Ophthalmology. 5th ed. Elsevier Health Sciences, Oxford, 2012.

10. Manesh AR, Mohebi N, Moghaddasi M. Assessment of retinal nerve fiber layer thickness in fellow eye in patients with unilateral optic neuritis. Arch Med 2016;8:4.

11. Miller PE, Galbreath EJ, Kehren JC, Steinberg H, Dubielzig RR. Photoreceptor cell death by apoptosis in dogs with sudden acquired retinal degeneration syndrome. Am J Vet Res 1998;59:149-152.

12. Muñana KR, Luttgen PJ. Prognostic factors for dogs with granulomatous meningoencephalomyelitis: 42 cases (19821996). J Am Vet Med Assoc 1998;212:1902-1906.

13. Nell B. Optic neuritis in dogs and cats. Vet Clin North Am Small Anim Pract 2008;38:403-415.

14. Osinchuk SC, Leis ML, Salpeter EM, Sandmeyer LS, Grahn BH. Evaluation of retinal morphology of canine sudden acquired retinal degeneration syndrome using optical coherence tomography and fluorescein angiography. Vet Ophthalmol 2018. Epub ahead of print. doi: 10.1111/vop.12602

15. Schwab S, Herden C, Seeliger F, Papaioannou N, Psalla D, Polizopulou Z, Baumgärtner W. Non-suppurative meningoencephalitis of unknown origin in cats and dogs: an immunohistochemical study. J Comp Pathol 2007;136:96-110.

16. Van der Woerdt A, Nasisse M, Davidson M. Sudden acquired retinal degeneration in the dog: clinical and laboratory findings in 36 cases. Prog Vet Comp Ophthalmol 1991;1:1118.

17. Zarfoss M, Schatzberg S, Venator K, Cutter-Schatzberg K, Cuddon P, Pintar J, Weinkle T, Scarlett J, Delahunta A. Combined cytosine arabinoside and prednisone therapy for meningoencephalitis of unknown aetiology in 10 dogs. J Small Anim Pract 2006;47:588-595. 